\title{
Non-Alcoholic Fatty Liver Disease and Its Association With Diabetes Mellitus
}

\author{
Jaskamal Padda $^{1}$, Khizer Khalid ${ }^{1}$, Anwar Khedr ${ }^{1}$, Fahriba Tasnim ${ }^{1}$, Ola A. Al-Ewaidat ${ }^{1}$, Ayden Charlene
} Cooper $^{1}$, Gutteridge Jean-Charles ${ }^{1,2}$

1. Internal Medicine, JC Medical Center, Orlando, USA 2. Internal Medicine, Advent Health \& Orlando Health Hospital, Orlando, USA

Corresponding author: Fahriba Tasnim, tasnimfahriba@gmail.com

\begin{abstract}
There is a bidirectional relationship between non-alcoholic fatty liver disease (NAFLD) and type 2 diabetes mellitus (T2DM). The liver has a vital role in the pathophysiology of both diseases as it leads to the development of insulin resistance (IR), which in turn results in NAFLD and T2DM. It has been shown that T2DM increases the risk of NAFLD progression. Furthermore, the presence of NAFLD raises the probability of T2DM complications, which explains the increased rates of NAFLD screening in patients with T2DM. In addition, there are common management options for the two diseases. Lifestyle changes can play a role in the initial management of both diseases. Medications that are used to treat T2DM are also used in the management of NAFLD, such as metformin, thiazolidinediones (TZD), glucagon-like peptide-1 (GLP-1) analogues, and dipeptidyl peptidase-4 (DPP4) inhibitors. Bariatric surgery is often used as a last resort and has shown promising results. Lifestyle interventions with diet and exercise are important postoperatively to maintain the weight loss. There are many novel treatments that are being investigated for the treatment of NAFLD, targeting multiple pathophysiologic pathways. This review aims to shed some light on the intricate relationship between NAFLD and T2DM and how IR links both diseases. We also try to raise awareness among clinicians about this relationship and how the presence of one disease should raise a high index of suspicion for the existence of the other.
\end{abstract}

Review began 08/11/2021 Review ended 08/16/2021 Published 08/20/2021

\section{() Copyright 2021}

Padda et al. This is an open access article distributed under the terms of the Creative Commons Attribution License CC-BY 4.0., which permits unrestricted use, distribution, and reproduction in any medium, provided the original author and source are credited.
Categories: Endocrinology/Diabetes/Metabolism, Internal Medicine, Gastroenterology Keywords: non-alcoholic fatty liver disease, diabetes mellitus, insulin resistance, oral hypoglycemics, hepatic steatosis, hepatocellular carcinoma, nash, bariatric surgery

\section{Introduction And Background}

Non-alcoholic fatty liver disease (NAFLD) is a range of liver disorders that includes hepatic steatosis, steatohepatitis, and hepatic fibrosis. This may further progress to cirrhosis and hepatocellular carcinoma (HCC) [1]. It is caused by fatty infiltration of more than $5 \%$ of liver hepatocytes, in the absence of a known cause of fatty liver disease, such as significant alcohol use, medications, or genetic disorders [2]. Approximately $25 \%$ of the global population has been affected by NAFLD [3]. Middle Eastern countries and South America account for the highest prevalence of NAFLD globally [4]. In the United States, it has been the major cause of chronic liver disease and around 90 million people are affected. Nearly one-quarter of them progresses to non-alcoholic steatohepatitis (NASH) [5]. The prevalence of NAFLD has been increasing progressively over the past years due to the higher prevalence of obesity, physical inactivity, metabolic syndromes, and type 2 diabetes mellitus (T2DM), which represent the major risk factors of NAFLD. It was found that more than half the patients with T2DM are diagnosed with NAFLD, which shows a strong relationship between them [6,7]. Furthermore, studies have shown that T2DM plays a major role in disease progression to NASH, fibrosis, and cirrhosis [8]. The majority of NAFLD patients are asymptomatic, and it is usually found incidentally during routine blood workups [9]. Although the gold standard diagnostic method for NAFLD is a liver biopsy, other, alternative, and non-invasive modalities have also been described, which include serum biomarkers and imaging studies. While ultrasound represents the main imaging study for NAFLD, CT and MRI have a diagnostic role as well [10]. However, reviewing the patient's history to exclude alcohol use and other causes of liver disease represents a major step in the diagnostic criteria of NAFLD [11].

\section{Review \\ Pathophysiology of NAFLD}

The Multi-Hit Theory

Although the pathogenesis of NAFLD has been investigated widely before, it is still not entirely understood. The most commonly proposed mechanism is the multi-hit theory. According to this, the first hit involves the build-up of triglycerides in the liver, causing steatosis. This increases the susceptibility of the liver to undergo second hits caused by oxidative stress, endoplasmic reticulum stress, mitochondrial dysfunction, inflammatory cytokines, adipokines, gut microbiota, and glucocorticoids [12,13]. The two-hit theory was modified to outline the role of free fatty acids (FFA) in causing liver injury through direct lipotoxicity. FFA 


\section{Cureus}

influx into the liver leads to lysosomal destabilization, activating inflammatory pathways such as the nuclear factor kappa B-dependent tumor necrosis factor-alpha pathway [14]. The third hit is proposed to be initiated by the death of hepatocytes. The progression from non-alcoholic fatty liver to NASH occurs when the protective mechanisms of FFA-mediated lipotoxicity become exhausted, and the rate of hepatocyte death exceeds the rate of hepatocyte regeneration. This triggers the activation of myofibroblasts, which produce liver progenitor cells. These cells induce inflammatory immune responses and differentiate to replace the dead hepatocytes, causing variable degrees of hepatic architecture distortion [15].

\section{The Role of Insulin Resistance}

The most important link between NAFLD and T2DM is insulin resistance (IR). IR is defined as a suboptimal response to insulin functions in different tissues [16]. Insulin-enhanced FFA transport to the liver, decreased B-oxidation of FFA, increased de novo lipogenesis due to activation of lipogenic enzymes via sterol receptorbinding protein 1c, and increased triglyceride synthesis through the Kennedy pathway arise from insulin's compromised capacity to regulate lipolysis in the liver, adipose tissue, and skeletal muscles in IR states [17]. In these states, the beta cells of the pancreas try to overcome it by secreting more insulin. Furthermore, insulin clearance is also suppressed in patients with T2DM. All of this results in hyperinsulinemia, which has been shown to cause hepatocellular ballooning and lobular inflammation [16]. IR is affected by many inflammatory pathways in NAFLD, which are summarized in Figure 1 [18].

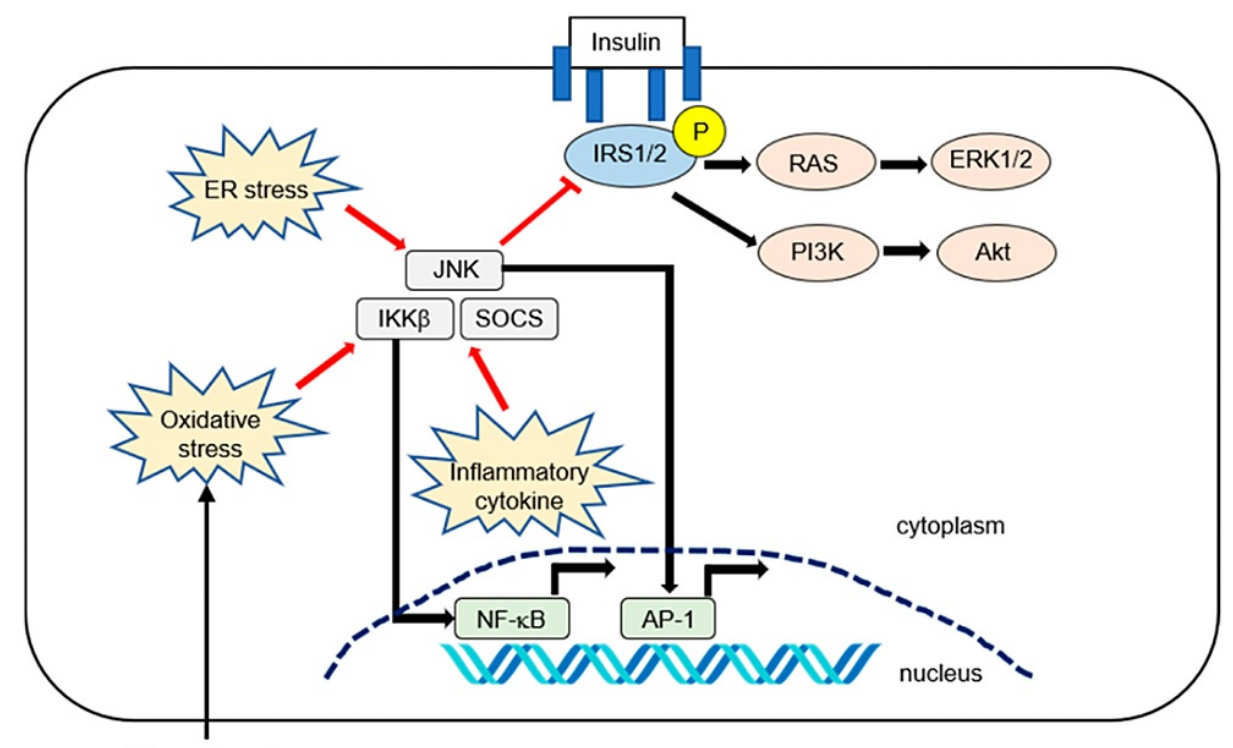

Hyperglicemia

\section{FIGURE 1: Summary of the inflammatory pathways affecting hepatic insulin resistance in NAFLD}

Insulin activates its receptor, which results in tyrosine phosphorylation of the IRS1 and IRS2 and activation of downstream effector pathways, including the PI3K-phosphoinositide-dependent kinase-AKT and the RASERK pathways. Pro-inflammatory signaling or reactive oxygen species can activate IKK- $\beta$. The activated NF$\mathrm{kB}$ is then translocated into the nucleus and binds to specific deoxyribonucleic acid response elements. Inflammatory cytokines such as interleukin-6 promote insulin resistance by inducing SOCS1 and SOCS3. SOCS1 and SOCS3 impair insulin signaling through ubiquitin-dependent degradation of IRS. The JNK, or mitogen-activated protein kinase, represents another important inhibitory kinase of IRS that is activated in response to a variety of extracellular stimuli and cellular stressors such as oxidative and ER stress

ER: endoplasmic reticulum; JNK: c-Jun N-terminal kinase; IKK- $\beta$ : IkappaB kinase beta; SOCS: suppressor of cytokine signaling; NF-KB: nuclear factor kappa-light-chain-enhancer of activated B cells; AP-1: activator protein 1; IRS1: insulin receptor substrate 1; IRS2: insulin receptor substrate 2; PI3K: phosphatidylinositol 3kinase; AKT: protein kinase B; RAS: rat sarcoma; ERK1/2: extracellular-signal-regulated kinase 1/2

Copyright/license: this figure is from an open-access article distributed under the terms and conditions of the Creative Commons Attribution (CC BY) license (http://creativecommons.org/licenses/by/4.0/). No modifications were made to the original figure

Fujii H, Kawada N, Japan Study Group Of Nafld J-N: The role of insulin resistance and diabetes in nonalcoholic fatty liver disease. Int J Mol Sci. 2020, 21:3863. 10.3390/ijms21113863 [18] 
Although there is a definite association between NAFLD and IR, it has been shown that there are some genetic variants that cause hepatic steatosis in the absence of IR. Primarily, the missense mutation isoleucine-to-methionine substitution at residue 148 within patatin-like phospholipase domain-containing protein 3 (PNPLA3) increases NAFLD progression and results in decreased hepatic triglyceride hydrolysis [19]. Another PNPLA3 mutation [PNPLA3 glutamic acid-to-lysine substitution at residue 434, single nucleotide polymorphisms (SNP) rs2294918] has an effect on the PNPLA3 phenotype by lowering hepatic messenger RNA and protein levels. The glutamic acid-to-lysine substitution at residue 167 variant (at SNP rs58542926) of the transmembrane 6 superfamily member 2 protein influences liver fat content. This variant has been linked to a $34 \%$ increase in liver fat level in its carriers. Lastly, a mutation in the gene encoding 1acylglycerol-3-phosphate O-acyltransferase causes its shortage, which results in severe steatosis [20].

\section{NAFLD increases the risk of diabetes mellitus (DM) incidence}

Studies have shown that NAFLD is a well-defined risk factor for T2DM. Various Korean observational studies have shown this correlation by tracking the risk of T2DM in non-DM patients over a five-year period based on their NAFLD status [21]. As NAFLD is a reversible condition in its early stages, it was found that the risk of T2DM decreased in patients with resolved NAFLD status [22]. In addition, studies have shown that the sustainability of NAFLD status is a key factor in increasing the risk of T2DM. Therefore, only patients with persistent NAFLD over a considerable period of time have shown a higher risk of T2DM development [23]. To date, three previously published meta-analysis studies have shown the increased risk of T2DM in patients with NAFLD diagnosed by different diagnostic modalities including liver enzymes and imaging studies [2426]. The most recent one was published in 2018, and in it, most NAFLD patients were diagnosed by imaging studies. It also showed that the severity of NAFLD is directly correlated to T2DM risk, which carries a future impact on the diagnosis and management of T2DM [26]. This study has been the most extensive and valuable in the literature, as imaging studies are considered to be more accurate and specific NAFLD diagnostic modalities than serum markers [27]. Although liver biopsy is still the gold standard diagnostic modality, there has been no meta-analysis of biopsy-diagnosed NAFLD due to the unavailability of such studies. Therefore, more efforts are needed to investigate this to prove the true causality between NAFLD and T2DM and to demonstrate the extent of T2DM risk caused by variable NAFLD stages. Furthermore, more studies are needed to be conducted on populations with diverse ethnicities, as the previous studies were done mostly on Asian populations [26]. Regardless of these limitations, the most recent recommendation is to screen patients with NAFLD for T2DM [27].

\section{DM increases the risk of NAFLD incidence}

T2DM is considered a well-known risk factor for NAFLD. Studies have shown a higher prevalence of NAFLD in DM patients [28]. A recent meta-analysis published in 2017 showed that the pooled prevalence of NAFLD in DM patients is around $60 \%$. This may vary according to gender, BMI, and the presence of other chronic disorders [29]. In addition, patients who presented with NAFLD and T2DM have shown a greater risk of chronic liver disease, fibrosis, and cirrhosis compared to non-DM patients [30] Moreover, NAFLD in DM patients increases the risk of cardiovascular disease and other diabetic complications [31]. Due to the progressively increasing incidence of T2DM over the past years [32], more studies have been conducted to assess the significance of early screening and treatment of NAFLD in DM patients [33]. Also, it has been suggested that there is a potential advantage in screening DM patients for NAFLD using MRI and magnetic resonance elastography (MRE) [34]. However, other studies support the importance of the healthcare providers to be watchful for NAFLD and chronic liver disease development in DM patients with no need for regular screening [35].

\section{NAFLD increases the risk of DM complications}

Several studies indicate that the effect of NAFLD is not confined to liver-related complications anymore. Moreover, it has a major influence on worsening DM complications. A study comprising 102 patients with NAFLD demonstrated that the incidence of impaired fasting glucose, dyslipidemia with elevated triglycerides and/or hypercholesterolemia, and hypertension increased after they had been diagnosed with NAFLD, necessitating regular screening for metabolic disorders in NAFLD patients [36]. Another study consisting of 358 patients with NAFLD and 788 matched controls with a follow-up period of six years showed evidence of increased incidence of obesity, hypertension, hypertriglyceridemia, hypercholesterolemia, DM, and multiple metabolic disorders in the patients' group compared to the healthy controls [37]. Moreover, several studies have indicated that NAFLD is closely linked to an increased risk of chronic vascular complications of DM [38]. In 2017, Guo et al. identified a heightened prevalence of carotid and lower limb atherosclerotic plaques to be associated with ultrasound-diagnosed NAFLD in people with T2DM [39]. Moreover, another study comprising individuals with T2DM established that the prevalence of vascular diseases is higher in individuals with NAFLD compared with healthy people [40]. A meta-analysis also identified the association between NAFLD and incident cardiovascular disease events [41]. When it comes to renal complications of DM, several studies have consistently revealed that NAFLD is linked to chronic kidney disease (CKD) [42]. A study including patients with T2DM revealed that the risk of CKD or advanced diabetic neuropathy increases twofold in patients with NAFLD independent of other factors [43]. In another study consisting of patients with T2DM, an association was found to be present between NAFLD and increased risk of incident CKD independent of other factors (adjusted hazard ratio: 1.49, 95\% CI: 1.1-2.2) [44]. Moreover, a cohort study revealed NAFLD to be associated with a nearly threefold increased risk of CKD 
even after adjusting for confounding variables [45]. On the other hand, several experiments indicate NAFLD to be linked to a heightened prevalence of distal symmetric polyneuropathy in individuals with type 1 diabetes mellitus (T1DM) or T2DM [46,47]. Although there have been multiple studies showing evidence of the worse impact of NAFLD in DM complications, further studies are needed to establish this relationship with more precision.

\section{DM increases the risk of NAFLD progression}

T2DM plays an important role in the progression of NAFLD as T2DM has been proven to augment the risk of NASH by approximately two to threefold [30]. Based on liver histology, studies reveal that about $80 \%$ of patients with T2DM exhibit NASH, and 30-40\% of them exhibit advanced fibrosis [48]. It was shown in a study that NAFLD and T2DM were established to be present in patients with HCC [49]. Furthermore, the risk of developing HCC increases by two to threefold with the presence of DM alone [50]. Therefore, the advancement of NAFLD is driven by the presence of DM. Numerous patients who have NASH developing into HCC have been found to have T2DM and other metabolic disorders [51,52]. Some researchers consider NAFLD to be an overlooked complication of DM due to the close association betweenT2DM and the progression of NAFLD to NASH [53].

DM augments NAFLD in many ways. Fatty-acid release from adipose tissue is increased by IR [54]. The IR status often plays a significant role in the upregulation of the hepatic uptake of fatty acids [55]. Liver inflammation and fibrosis are induced by direct lipotoxicity caused by excessive hepatic FFA influx in DM patients $[56,57]$. Further damage is caused to the liver by the oxidative stress induced by oxidation and metabolism of excessive FFAs in the liver [58], which consequently elicits hepatocellular damage and apoptosis. Hepatocyte apoptosis and necrosis are then activated by hepatocellular injury [59]. Consequently, this causes stimulation of hepatic stellate cells, and ultimately hepatic fibrosis [60]. There has been further evidence that liver fibrosis can be promoted by IR because of the stimulation of lysyl oxidase-like 2 , independent of hepatic stellate cell activity [61].

\section{Diagnosis and screening}

Diagnosis of NAFLD

The diagnosis of NAFLD is made when there is evidence of hepatic steatosis on imaging or biopsy, with the exclusion of significant alcohol consumption, and no other etiologies for hepatic steatosis or chronic liver diseases. NAFLD is usually diagnosed incidentally during testing or screening for other diseases, on finding abnormal liver enzymes or abnormal abdominal imaging outcomes [62,63]. However, a liver biopsy is required to reach a definitive diagnosis of NAFLD and also for grading and staging of the disease [64]. There are many investigative modalities that have been studied to either diagnose NAFLD or monitor its severity, including blood tests, imaging studies, liver biopsy, and clinical scoring systems.

Blood tests such as liver enzymes are not accurate biomarkers of NAFLD. Even though they can be the only abnormal findings in NAFLD patients, up to $80 \%$ of NAFLD patients can have normal liver enzymes [65]. Furthermore, it was found that histological findings are similar in patients with NAFLD with normal alanine aminotransferase (ALT) levels when compared to those with high ALT levels [66]. It was also found that $76 \%$ of patients with DM and normal ALT levels have a high prevalence of NAFLD [30]. Novel diagnostic markers are being studied to diagnose NAFLD, such as microRNA 22, with promising results so far [67]. Several other biomarkers have been studied to help with fibrosis staging in NAFLD. For example, it was found that elevated immunoglobulin A levels are found in NAFLD patients and can predict the progression to an advanced fibrosis stage [68]. In addition, clinical scoring systems such as the NAFLD fibrosis score can be used to identify patients with NAFLD who are at a high risk of advanced disease and require further assessment [69].

Ultrasound is recommended as the first-line imaging tool for diagnosing NAFLD [64] because of its ability to assess hepatic fat content (Table 1) [70,71]. Due to its safety profile, low cost, accessibility, and reliability, it is recommended as the imaging technique of choice for screening for fatty liver [72]. Another imaging technique is the controlled attenuation parameter (CAP) (Table 2) [73]. It is an ultrasound-based elastography technique used to detect the severity of liver steatosis. CAP has been found to have good sensitivity and specificity, but its diagnostic accuracy is questionable, according to a meta-analysis by Shi et al. [74]. CT can also be used as an initial technique to diagnose NAFLD [63]. However, there are certain limitations to CT, such as its high cost, radiation exposure, non-availability for routine use, and low sensitivity in detecting mildly elevated hepatic fat content [70]. Other imaging options are MRI-proton density fat fraction (MRI-PDFF) and magnetic reasoning spectrometry (MRS). MRI-PDFF has been found to correlate with the steatosis stage accurately, but it is suboptimal in detecting advanced fibrosis stages [75]. MRS is not recommended for use in the clinical setting [64], and its use is limited to research due to high cost, reduced accessibility, and the need for additional validation [76]. There are other imaging techniques that are used to diagnose fibrosis in NAFLD, such as transient elastography (FibroScan, Echosens ${ }^{\mathrm{TM}}$, Paris, France), MRE, and acoustic radiation force impulse imaging [63]. 


\section{Cureus}

\begin{tabular}{|l|l|l|l|}
\hline Score & Steatosis & $\begin{array}{l}\text { Description of fatty infiltration in the } \\
\text { liver }\end{array}$ & Visualization \\
\hline 0 & Absent & $\begin{array}{l}\text { Normal echotexture of the liver } \\
\text { Slight/diffuse increase in liver }\end{array}$ & Normal visualization of the portal vein wall \\
\hline 1 & Mild & $\begin{array}{l}\text { Normal visualization of the portal vein wall } \\
\text { echogenicity }\end{array}$ \\
\hline 2 & Moderate & Increase in liver echogenicity & $\begin{array}{l}\text { Slightly impaired appearance of the portal vein wall } \\
\text { Poor/no visualization of the portal vein wall and posterior part of the }\end{array}$ \\
\hline 3 & Severe & Increase in liver echogenicity & \begin{tabular}{l} 
right liver lobe \\
\hline
\end{tabular}
\end{tabular}

\section{TABLE 1: Steatosis grading via ultrasound}

Ultrasound grading with descriptions of liver parenchyma and vessel appearance [71]

\begin{tabular}{|c|c|c|}
\hline Grade & CAP score & Fatty changes in the liver \\
\hline S0: no steatosis & $0-237$ dB/m & $0-10 \%$ \\
\hline S1: mild steatosis & $238-259$ dB/m & $11-33 \%$ \\
\hline S2: moderate steatosis & $260-292 \mathrm{~dB} / \mathrm{m}$ & $34-66 \%$ \\
\hline S3: severe steatosis & $>293 \mathrm{~dB} / \mathrm{m}$ & $>67 \%$ \\
\hline
\end{tabular}

\section{TABLE 2: CAP score}

CAP: controlled attenuation parameter; $\mathrm{dB} / \mathrm{m}$ : decibels per meter [73]

There has been much debate about the routine use of liver biopsy for diagnosing NAFLD, considering its invasiveness, even though it remains the gold standard of diagnosis. It is recommended for confirming the diagnosis of NASH, monitoring patients with a high risk of disease progression, persistent elevation of liver enzymes for more than six months, excluding other causes of chronic liver diseases, and inconclusive imaging $[63,64]$. To diagnose NASH, three main histologic features are required: steatosis, lobular inflammation, and hepatocellular ballooning [64].

\section{Should We Screen for NAFLD in Patients with T2DM?}

Because of the high prevalence of NAFLD in patients with T2DM, the presence of T2DM should raise high suspicion for the simultaneous existence of NAFLD [77]. However, the American Gastroenterological Association does not recommend routine screening of NAFLD in patients with T2DM due to concerns pertaining to long-term benefits and cost-effectiveness [62]. There have been many studies that showed the benefits of screening for NAFLD in patients with T2DM. For example, in a prospective cross-sectional study, 679 patients with a confirmed diagnosis of T2DM were screened for NAFLD using transient elastography, and the findings showed a prevalence of $83.6 \%$ of NAFLD. In light of this, the authors suggested that there is an urgent need for NAFLD screening in patients with T2DM [78].

\section{The role of lifestyle changes in the management of NAFLD and T2DM}

Studies have shown a reciprocal relationship between T2DM and NAFLD, which means that they both can lead to each other [79]. In addition, T2DM and NAFLD share the same risk factors, which are mostly related to lifestyle habits. As a result, lifestyle changes have been considered as the first-line step in the prevention and treatment of NAFLD and T2DM. These lifestyle changes include diet, exercise, and weight loss [80]. With the progressively increasing incidence of NAFLD and T2DM over the past years, they have been considered a major risk factor for chronic liver disease and cardiovascular diseases. Thus, more efforts have been made to determine the exact effect of lifestyle changes on disease progression [81]. Diet changes include a low caloric diet in terms of carbohydrate and fat restriction [82]. Studies have shown that weight loss caused by a low carbohydrate diet has a more significant effect on NAFLD regression than a low caloric diet [83]. In addition, it has been shown that polyunsaturated fatty acids have a protecting effect on NAFLD [82]. However, high protein intake increases the risk of NAFLD [84]. Weight loss has a significant role in NAFLD and T2DM treatment. More than $5 \%$ weight loss is needed to reverse liver steatosis and around $10 \%$ is required for 
NASH regression [85]. Regarding physical activity, studies have shown that increased physical activity is associated with a remarkable improvement in liver function and other metabolic markers of the body, regardless of the patient's weight-loss status [86].

\section{The role of oral hypoglycemics in the management of DM and how they affect the management of NAFLD}

The main pathogenic mechanism of NAFLD is thought to be impaired response to insulin actions, or IR that leads to high amounts of FFAs and glucose in the blood, which consequently leads to accumulation of liver fat. For this reason, several experiments have been undertaken on the use of anti-diabetic drugs in patients with NAFLD [87].

\section{Metformin}

Four meta-analyses [88-91] were performed on various studies on the use of metformin in NAFLD. Although it demonstrated no major progress in liver histology, in the case of liver fibrosis, it revealed substantial improvement in blood cholesterol level, fasting plasma glucose, and hemoglobin A1c (HbA1c). These findings suggest that metformin can be useful as a treatment against NAFLD risk factors. Although the impact of NAFLD and IR on cancer development is widely recognized, metformin use appears to have a protective function against both hepatic [92,93] and non-hepatic cancers [94].

Thiazolidinediones (TZD)

Several meta-analyses [95-98] have been conducted to determine the role of TZD on liver histology in NASH patients, and the results have varied. All of them showed beneficial effects of TZD on lobular inflammation without any substantial improvement in the fibrosis of the liver. However, adding lifestyle modification to TZD yielded considerable improvement.

Glucagon-like Peptide-1 (GLP-1) Analogues

In an experiment where animals were treated with exenatide, a reduction in hepatic fat was identified [99101]. However, whether human hepatocytes have GLP-1 receptors or how GLP-1 agonists act on the liver is still not clear [99-102]. According to the liraglutide safety and efficacy in patients with non-alcoholic steatohepatitis (LEAN) trial [103], liraglutide appreciably improved steatosis and hepatocyte ballooning, but there were no significant differences in either lobular inflammation or NAFLD Activity Score.

Sodium-Glucose Cotransporter 2 Inhibitors

Several pre-clinical studies performed with animal experimental models have demonstrated that canagliflozin, empagliflozin, luseogliflozin, tofogliflozin, ipragliflozin, and remogliflozin [104-112] could be linked to hepatic steatosis improvement. Moreover, another finding has stated that tofogliflozin possibly decreases the risk of advancement to HCC.

Dipeptidyl Peptidase-4 (DPP4) Inhibitors

A report in 2012 was the first to show an improvement in glycosylated hemoglobin as well as in hepatic steatosis on MRI in a 67-year-old Asian woman diagnosed with NAFLD who was treated with sitagliptin [113]. Furthermore, a randomized-controlled trial comprising 58 patients, who were randomly assigned to receive either placebo or vildagliptin $50 \mathrm{mg}$ twice a day for 12 weeks, demonstrated significant improvement in BMI, cholesterol, aminotransferase, and triglyceride levels among those who took vildagliptin [114].

Sulfonylureas and Meglitinides

Sulfonylureas are typically contraindicated in chronic liver or renal disease patients due to their hepatic metabolism and renal excretion [115]. Meglitinides have a shorter half-life than sulfonylureas and they do not have considerable renal excretion. But it is still not clear whether repaglinide is safe to be used in DM patients with chronic liver disease [116,117].

\section{The role of bariatric surgery in the management of NAFLD and T2DM}

Bariatric surgery is often considered the last option in the treatment of obesity-related health conditions. Lifestyle interventions with diet and exercise are important postoperatively to maintain the weight loss [118]. Questionnaires can be used to assess adherence to diet and exercise. A questionnaire developed by Dubai et al. consisting of 14 questions proved to be a reliable tool to gauge adherence to lifestyle modification in 100 obese patients with NAFLD [119]. A number of studies have shown the positive impact of bariatric surgery in DM and NAFLD. The combination of Roux-en-Y gastric bypass (RYGB) and optimal medicine has been shown to improve HbA1c postoperatively [120,121]. After two years, patients who 
underwent RYGB had lower levels of fasting glucose compared to patients who received only medical therapy [120]. Patients undergoing vertical sleeve gastrectomy also had similar findings [121]. Moreover, once patients lose weight after bariatric surgery, IR gets improved, and it seems to correlate with improved Creactive protein and tumor necrosis factor- $\alpha$ [122]. Additionally, there is also improvement in kidney damage as demonstrated by improved microalbuminuria [123].

There have also been significantly favorable outcomes when bariatric surgery was considered for proper candidates with NAFLD who have been unable to achieve adequate weight reduction by diet and lifestyle changes only. In a meta-analysis comprising 15 studies, $91 \%$ of patients had improvement in steatosis and $65.5 \%$ had decreased fibrosis [124]. Approximately $70 \%$ of patients had a total resolution of NASH. Most of these studies were prospective and utilized RYGB as the method of bariatric surgery. On the other hand, a study comprising seven morbidly obese patients with NAFLD demonstrated improvement in transforming growth factor- $\beta 1, \alpha$-smooth muscle actin, and inflammatory markers such as interleukin-8, which are considered as hepatic factors regulating fibrogenesis [125]. Several studies consisting of morbidly obese NAFLD patients have revealed positive outcomes with bariatric surgery: reduction in steatosis, lobular inflammation, ballooning, and fibrosis [126,127]. However, when it comes to the comparison between the efficacy of different types of bariatric surgery, RYGB appears to be superior to all others due to its excellent weight-loss effects [128]. In another one-year cohort study including morbidly obese NASH patients, $85 \%$ of patients had NASH resolution overall, but greater rates were accomplished after RYGB compared to adjustable gastric band (AGB) surgery [129]. Moreover, patients undergoing AGB had greater rates of persisting NASH compared to those who had RYGB [129].

\section{Emerging treatments for NAFLD}

Due to the many complex pathways involved in the pathogenesis of NAFLD, several therapeutic options have been investigated. In Table 3, we summarize some of the most significant interventional clinical trials with published results, which have tested therapies for NAFLD in adult patients [130-132].

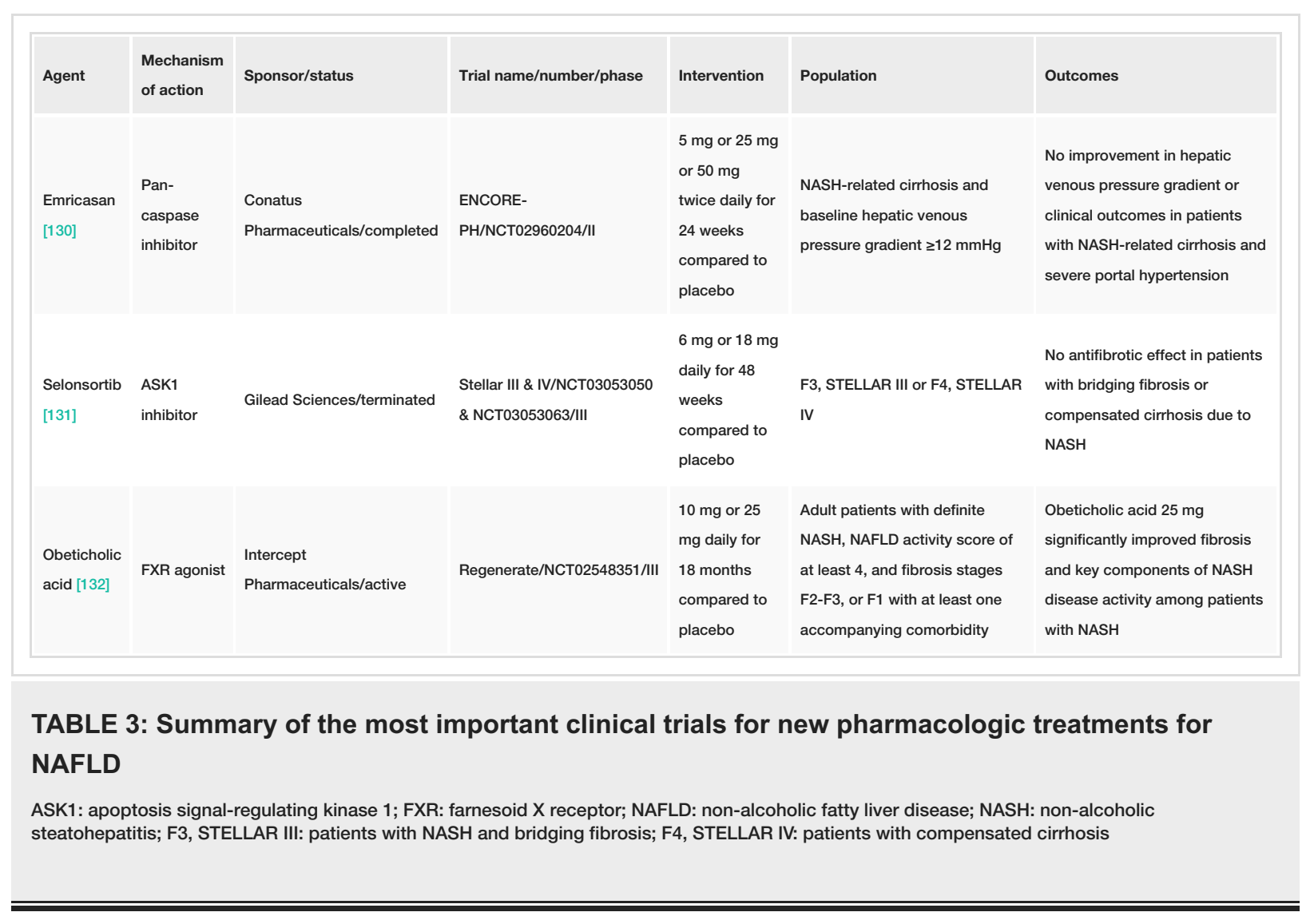

\section{Conclusions}

NAFLD and T2DM are considered as two different manifestations of the same pathology that is IR. The majority of chronic liver disease cases are caused by NAFLD. Due to its strong association with T2DM, it has been a hot research topic to further explore this relationship and its future clinical implications. NAFLD and T2DM are both considered risk factors for each other. This makes us attentive to the potential impact of early screening of one disease in the previous presence of the other. In addition, the coexistence of NAFLD and T2DM has synergistic effects on each disease's progression and complications. NAFLD and T2DM have the same pathophysiology and almost the same risk factors. Therefore, they share the same prevention and 
management strategies, which mainly consist of lifestyle changes, the use of oral hypoglycemics, and bariatric surgery as the last-resort treatment modality, which has shown its effective impact on NAFLD and T2DM management. However, there have also been efforts to find novel treatment modalities for NAFLD, such as FXR agonists, ASK1 inhibitors, and pan-caspase inhibitors.

\section{Additional Information \\ Disclosures}

Conflicts of interest: In compliance with the ICMJE uniform disclosure form, all authors declare the following: Payment/services info: All authors have declared that no financial support was received from any organization for the submitted work. Financial relationships: All authors have declared that they have no financial relationships at present or within the previous three years with any organizations that might have an interest in the submitted work. Other relationships: All authors have declared that there are no other relationships or activities that could appear to have influenced the submitted work.

\section{References}

1. Smith BW, Adams LA: Non-alcoholic fatty liver disease. Crit Rev Clin Lab Sci. 2011, 48:97-113. 10.3109/10408363.2011.596521

2. Tomic D, Kemp WW, Roberts SK: Nonalcoholic fatty liver disease: current concepts, epidemiology and management strategies. Eur J Gastroenterol Hepatol. 2018, 30:1103-15. 10.1097/MEG.0000000000001235

3. Huang DQ, El-Serag HB, Loomba R: Global epidemiology of NAFLD-related HCC: trends, predictions, risk factors and prevention. Nat Rev Gastroenterol Hepatol. 2021, 18:223-38. 10.1038/s41575-020-00381-6

4. Mitra S, De A, Chowdhury A: Epidemiology of non-alcoholic and alcoholic fatty liver diseases. Transl Gastroenterol Hepatol. 2020, 5:16. 10.21037/tgh.2019.09.08

5. Perumpail BJ, Khan MA, Yoo ER, Cholankeril G, Kim D, Ahmed A: Clinical epidemiology and disease burden of nonalcoholic fatty liver disease. World J Gastroenterol. 2017, 23:8263-76. 10.3748/wjg.v23.i47.8263

6. Younossi ZM, Stepanova M, Afendy M, Fang Y, Younossi Y, Mir H, Srishord M: Changes in the prevalence of the most common causes of chronic liver diseases in the United States from 1988 to 2008. Clin Gastroenterol Hepatol. 2011, 9:524-30. 10.1016/j.cgh.2011.03.020

7. Qiu S, Cai X, Sun Z, Li L, Zügel M, Steinacker JM, Schumann U: Association between physical activity and risk of nonalcoholic fatty liver disease: a meta-analysis. Therap Adv Gastroenterol. 2017, 10:701-13. 10.1177/1756283X17725977

8. Targher G, Corey KE, Byrne CD, Roden M: The complex link between NAFLD and type 2 diabetes mellitus mechanisms and treatments (Epub ahead of print). Nat Rev Gastroenterol Hepatol. 2021, 10.1038/s41575021-00448-y

9. Ahmed M: Non-alcoholic fatty liver disease in 2015. World J Hepatol. 2015, 7:1450-9. 10.4254/wjh.v7.i11.1450

10. Papatheodoridi M, Cholongitas E: Diagnosis of non-alcoholic fatty liver disease (NAFLD): current concepts . Curr Pharm Des. 2018, 24:4574-86. 10.2174/1381612825666190117102111

11. Hashimoto E, Taniai M, Tokushige K: Characteristics and diagnosis of NAFLD/NASH. J Gastroenterol Hepatol. 2013, 28:64-70. 10.1111/igh.12271

12. Dowman JK, Tomlinson JW, Newsome PN: Pathogenesis of non-alcoholic fatty liver disease . QJM. 2010, 103:71-83. 10.1093/qjmed/hcp158

13. Hazlehurst JM, Woods C, Marjot T, Cobbold JF, Tomlinson JW: Non-alcoholic fatty liver disease and diabetes. Metabolism. 2016, 65:1096-108. 10.1016/j.metabol.2016.01.001

14. Feldstein AE, Werneburg NW, Canbay A, et al.: Free fatty acids promote hepatic lipotoxicity by stimulating TNF-alpha expression via a lysosomal pathway. Hepatology. 2004, 40:185-94. 10.1002/hep.20283

15. Jou J, Choi SS, Diehl AM: Mechanisms of disease progression in nonalcoholic fatty liver disease . Semin Liver Dis. 2008, 28:370-9. 10.1055/s-0028-1091981

16. Tanase DM, Gosav EM, Costea CF, et al.: The intricate relationship between type 2 diabetes mellitus (T2DM), insulin resistance (IR), and nonalcoholic fatty liver disease (NAFLD). J Diabetes Res. 2020, 2020:3920196. 10.1155/2020/3920196

17. Watt MJ, Miotto PM, De Nardo W, Montgomery MK: The liver as an endocrine organ-linking NAFLD and insulin resistance. Endocr Rev. 2019, 40:1367-93. 10.1210/er.2019-00034

18. Fujii H, Kawada N, Japan Study Group Of Nafld Jsg-Nafld: The role of insulin resistance and diabetes in nonalcoholic fatty liver disease. Int J Mol Sci. 2020, 21:3863. 10.3390/ijms21113863

19. Kantartzis K, Peter A, Machicao F, et al.: Dissociation between fatty liver and insulin resistance in humans carrying a variant of the patatin-like phospholipase 3 gene. Diabetes. 2009, 58:2616-23. 10.2337/db09-0279

20. Tilg H, Moschen AR, Roden M: NAFLD and diabetes mellitus. Nat Rev Gastroenterol Hepatol. 2017, 14:3242. 10.1038/nrgastro.2016.147

21. Rhee EJ: Nonalcoholic fatty liver disease and diabetes: an epidemiological perspective . Endocrinol Metab (Seoul). 2019, 34:226-33. 10.3803/EnM.2019.34.3.226

22. Sung KC, Wild SH, Byrne CD: Resolution of fatty liver and risk of incident diabetes . J Clin Endocrinol Metab. 2013, 98:3637-43. 10.1210/jc.2013-1519

23. Bae JC, Han JM, Cho JH, et al.: The persistence of fatty liver has a differential impact on the development of diabetes: The Kangbuk Samsung Health Study. Diabetes Res Clin Pract. 2018, 135:1-6. 10.1016/i.diabres.2017.10.019

24. Musso G, Gambino R, Cassader M, Pagano G: Meta-analysis: natural history of non-alcoholic fatty liver disease (NAFLD) and diagnostic accuracy of non-invasive tests for liver disease severity. Ann Med. 2011, 43:617-49. 10.3109/07853890.2010.518623

25. Ballestri S, Zona S, Targher G, et al.: Nonalcoholic fatty liver disease is associated with an almost twofold 
increased risk of incident type 2 diabetes and metabolic syndrome. Evidence from a systematic review and meta-analysis. J Gastroenterol Hepatol. 2016, 31:936-44. 10.1111/igh.13264

26. Mantovani A, Byrne CD, Bonora E, Targher G: Nonalcoholic fatty liver disease and risk of incident type 2 diabetes: a meta-analysis. Diabetes Care. 2018, 41:372-82. 10.2337/dc17-1902

27. Papagianni M, Sofogianni A, Tziomalos K: Non-invasive methods for the diagnosis of nonalcoholic fatty liver disease. World J Hepatol. 2015, 7:638-48. 10.4254/wjh.v7.i4.638

28. Yi M, Chen RP, Yang R, Chen H: Increased prevalence and risk of non-alcoholic fatty liver disease in overweight and obese patients with type 2 diabetes in South China. Diabet Med. 2017, 34:505-13. 10.1111/dme.13174

29. Dai W, Ye L, Liu A, Wen SW, Deng J, Wu X, Lai Z: Prevalence of nonalcoholic fatty liver disease in patients with type 2 diabetes mellitus: a meta-analysis. Medicine (Baltimore). 2017, 96:e8179. 10.1097/MD.0000000000008179

30. Portillo-Sanchez P, Bril F, Maximos M, et al.: High prevalence of nonalcoholic fatty liver disease in patients with type 2 diabetes mellitus and normal plasma aminotransferase levels. J Clin Endocrinol Metab. 2015, 100:2231-8. 10.1210/jc.2015-1966

31. Targher G, Bertolini L, Rodella S, Tessari R, Zenari L, Lippi G, Arcaro G: Nonalcoholic fatty liver disease is independently associated with an increased incidence of cardiovascular events in type 2 diabetic patients. Diabetes Care. 2007, 30:2119-21. 10.2337/dc07-0349

32. Wu Y, Ding Y, Tanaka Y, Zhang W: Risk factors contributing to type 2 diabetes and recent advances in the treatment and prevention. Int J Med Sci. 2014, 11:1185-200. 10.7150/ijms.10001

33. Pandyarajan V, Gish RG, Alkhouri N, Noureddin M: Screening for nonalcoholic fatty liver disease in the primary care clinic. Gastroenterol Hepatol (N Y). 2019, 15:357-65.

34. Doycheva I, Cui J, Nguyen P, et al.: Non-invasive screening of diabetics in primary care for NAFLD and advanced fibrosis by MRI and MRE. Aliment Pharmacol Ther. 2016, 43:83-95. 10.1111/apt.13405

35. Wong VW, Chalasani N: Not routine screening, but vigilance for chronic liver disease in patients with type 2 diabetes. J Hepatol. 2016, 64:1211-3. 10.1016/j.jhep.2016.02.032

36. Friis-Liby I, Aldenborg F, Jerlstad P, Rundström K, Björnsson E: High prevalence of metabolic complications in patients with non-alcoholic fatty liver disease. Scand J Gastroenterol. 2004, 39:864-9. 10.1080/00365520410006431

37. Fan JG, Li F, Cai XB, Peng YD, Ao QH, Gao Y: Effects of nonalcoholic fatty liver disease on the development of metabolic disorders. J Gastroenterol Hepatol. 2007, 22:1086-91. 10.1111/j.1440-1746.2006.04781.x

38. Targher G, Byrne CD: Clinical Review: nonalcoholic fatty liver disease: a novel cardiometabolic risk factor for type 2 diabetes and its complications. J Clin Endocrinol Metab. 2013, 98:483-95. 10.1210/jc.2012-3093

39. Guo K, Zhang L, Lu J, et al.: Non-alcoholic fatty liver disease is associated with late but not early atherosclerotic lesions in Chinese inpatients with type 2 diabetes. J Diabetes Complications. 2017, 31:80-5. 10.1016/j.jdiacomp.2016.09.008

40. Targher G, Bertolini L, Padovani R, et al.: Prevalence of nonalcoholic fatty liver disease and its association with cardiovascular disease among type 2 diabetic patients. Diabetes Care. 2007, 30:1212-8. 10.2337/dc062247

41. Targher G, Byrne CD, Lonardo A, Zoppini G, Barbui C: Non-alcoholic fatty liver disease and risk of incident cardiovascular disease: a meta-analysis. J Hepatol. 2016, 65:589-600. 10.1016/j.jhep.2016.05.013

42. Targher G, Chonchol MB, Byrne CD: CKD and nonalcoholic fatty liver disease. Am J Kidney Dis. 2014, 64:638-52. 10.1053/j.ajkd.2014.05.019

43. Targher G, Bertolini L, Rodella S, Zoppini G, Lippi G, Day C, Muggeo M: Non-alcoholic fatty liver disease is independently associated with an increased prevalence of chronic kidney disease and proliferative/lasertreated retinopathy in type 2 diabetic patients. Diabetologia. 2008, 51:444-50. 10.1007/s00125-007-0897-4

44. Targher $\mathrm{G}$, Chonchol M, Bertolini L, et al.: Increased risk of CKD among type 2 diabetics with nonalcoholic fatty liver disease. J Am Soc Nephrol. 2008, 19:1564-70. 10.1681/ASN.2007101155

45. Targher G, Mantovani A, Pichiri I, et al.: Nonalcoholic fatty liver disease is independently associated with an increased incidence of chronic kidney disease in patients with type 1 diabetes. Diabetes Care. 2014, 37:1729-36. 10.2337/dc13-2704

46. Mantovani A, Rigolon R, Mingolla L, et al.: Nonalcoholic fatty liver disease is associated with an increased prevalence of distal symmetric polyneuropathy in adult patients with type 1 diabetes. J Diabetes Complications. 2017, 31:1021-6. 10.1016/j.jdiacomp.2017.01.024

47. Williams KH, Burns K, Constantino M, et al.: An association of large-fibre peripheral nerve dysfunction with non-invasive measures of liver fibrosis secondary to non-alcoholic fatty liver disease in diabetes. J Diabetes Complications. 2015, 29:1240-7. 10.1016/j.jdiacomp.2015.06.015

48. Bazick J, Donithan M, Neuschwander-Tetri BA, et al.: Clinical model for NASH and advanced fibrosis in adult patients with diabetes and NAFLD: guidelines for referral in NAFLD. Diabetes Care. 2015, 38:1347-55. 10.2337/dc14-1239

49. Sanyal A, Poklepovic A, Moyneur E, Barghout V: Population-based risk factors and resource utilization for HCC: US perspective. Curr Med Res Opin. 2010, 26:2183-91. 10.1185/03007995.2010.506375

50. El-Serag HB, Tran T, Everhart JE: Diabetes increases the risk of chronic liver disease and hepatocellular carcinoma. Gastroenterology. 2004, 126:460-8. 10.1053/j.gastro.2003.10.065

51. Yasui K, Hashimoto E, Komorizono Y, et al.: Characteristics of patients with nonalcoholic steatohepatitis who develop hepatocellular carcinoma. Clin Gastroenterol Hepatol. 2011, 9:428-33. 10.1016/j.cgh.2011.01.023

52. Aleksandrova K, Boeing $\mathrm{H}$, Nöthlings $\mathrm{U}$, et al.: Inflammatory and metabolic biomarkers and risk of liver and biliary tract cancer. Hepatology. 2014, 60:858-71. 10.1002/hep.27016

53. Arrese M: Nonalcoholic fatty liver disease: liver disease: an overlooked complication of diabetes mellitus . Nat Rev Endocrinol. 2010, 6:660-1. 10.1038/nrendo.2010.173

54. Kim DS, Jackson AU, Li YK, et al.: Novel association of TM6SF2 rs58542926 genotype with increased serum tyrosine levels and decreased apoB-100 particles in Finns. J Lipid Res. 2017, 58:1471-81.

10.1194/jlr.P076034 
55. Miquilena-Colina ME, Lima-Cabello E, Sánchez-Campos S, et al.: Hepatic fatty acid translocase CD36 upregulation is associated with insulin resistance, hyperinsulinaemia and increased steatosis in nonalcoholic steatohepatitis and chronic hepatitis C. Gut. 2011, 60:1394-402. 10.1136/gut.2010.222844

56. Tomita K, Teratani T, Suzuki T, et al.: Free cholesterol accumulation in hepatic stellate cells: mechanism of liver fibrosis aggravation in nonalcoholic steatohepatitis in mice. Hepatology. 2014, 59:154-69. 10.1002/hep.26604

57. Marra F, Svegliati-Baroni G: Lipotoxicity and the gut-liver axis in NASH pathogenesis . J Hepatol. 2018, 68:280-95. 10.1016/j.jhep.2017.11.014

58. Paradies G, Paradies V, Ruggiero FM, Petrosillo G: Oxidative stress, cardiolipin and mitochondrial dysfunction in nonalcoholic fatty liver disease. World J Gastroenterol. 2014, 20:14205-18. 10.3748/wjg.v20.i39.14205

59. Luedde T, Kaplowitz N, Schwabe RF: Cell death and cell death responses in liver disease: mechanisms and clinical relevance. Gastroenterology. 2014, 147:765-83. 10.1053/j.gastro.2014.07.018

60. Wree A, Eguchi A, McGeough MD, et al.: NLRP3 inflammasome activation results in hepatocyte pyroptosis, liver inflammation, and fibrosis in mice. Hepatology. 2014, 59:898-910. 10.1002/hep.26592

61. Dongiovanni P, Meroni M, Baselli GA, et al.: Insulin resistance promotes Lysyl Oxidase Like 2 induction and fibrosis accumulation in non-alcoholic fatty liver disease. Clin Sci (Lond). 2017, 131:1301-15. 10.1042/CS20170175

62. Chalasani N, Younossi Z, Lavine JE, et al.: The diagnosis and management of non-alcoholic fatty liver disease: practice guideline by the American Gastroenterological Association, American Association for the Study of Liver Diseases, and American College of Gastroenterology. Gastroenterology. 2012, 142:1592-609. 10.1053/j.gastro.2012.04.001

63. Rinella ME: Nonalcoholic fatty liver disease: a systematic review . JAMA. 2015, 313:2263-73. 10.1001/jama.2015.5370

64. European Association for the Study of the Liver (EASL); European Association for the Study of Diabetes (EASD); European Association for the Study of Obesity (EASO): EASL-EASD-EASO Clinical Practice Guidelines for the management of non-alcoholic fatty liver disease. J Hepatol. 2016, 64:1388-402. 10.1016/j.jhep.2015.11.004

65. Piazzolla VA, Mangia A: Noninvasive diagnosis of NAFLD and NASH. Cells. 2020, 9:1005. 10.3390/cells 9041005

66. Fracanzani AL, Valenti L, Bugianesi E, et al.: Risk of severe liver disease in nonalcoholic fatty liver disease with normal aminotransferase levels: a role for insulin resistance and diabetes. Hepatology. 2008, 48:792-8. 10.1002/hep.22429

67. Cai C, Lin Y, Yu C: Circulating miRNAs as novel diagnostic biomarkers in nonalcoholic fatty liver disease: a systematic review and meta-analysis. Can J Gastroenterol Hepatol. 2019, 2019:2096161. $10.1155 / 2019 / 2096161$

68. McPherson S, Henderson E, Burt AD, Day CP, Anstee QM: Serum immunoglobulin levels predict fibrosis in patients with non-alcoholic fatty liver disease. J Hepatol. 2014, 60:1055-62. 10.1016/j.jhep.2014.01.010

69. Osayande AS, Kale N: Nonalcoholic Fatty Liver Disease: Identifying Patients at Risk of Inflammation or Fibrosis. Am Fam Physician. 2017, 95:796-7.

70. Shen FF, Lu LG: Advances in noninvasive methods for diagnosing nonalcoholic fatty liver disease . J Dig Dis. 2016, 17:565-71. 10.1111/1751-2980.12384

71. Ferraioli G, Soares Monteiro LB: Ultrasound-based techniques for the diagnosis of liver steatosis . World J Gastroenterol. 2019, 25:6053-62. 10.3748/wig.v25.i40.6053

72. Hernaez R, Lazo M, Bonekamp S, Kamel I, Brancati FL, Guallar E, Clark JM: Diagnostic accuracy and reliability of ultrasonography for the detection of fatty liver: a meta-analysis. Hepatology. 2011, 54:1082-90. 10.1002/hep. 24452

73. Sansom SE, Martin J, Adeyemi O, et al.: Steatosis rates by liver biopsy and transient elastography with controlled attenuation parameter in clinical experience of hepatitis $\mathrm{C}$ virus (HCV) and human immunodeficiency virus/HCV coinfection in a large US hepatitis clinic. Open Forum Infect Dis. 2019, 6:ofz099. 10.1093/ofid/ofz099

74. Shi KQ, Tang JZ, Zhu XL, et al.: Controlled attenuation parameter for the detection of steatosis severity in chronic liver disease: a meta-analysis of diagnostic accuracy. J Gastroenterol Hepatol. 2014, 29:1149-58. 10.1111/jgh.12519

75. Wildman-Tobriner B, Middleton MM, Moylan CA, et al.: Association between magnetic resonance imagingproton density fat fraction and liver histology features in patients with nonalcoholic fatty liver disease or nonalcoholic steatohepatitis. Gastroenterology. 2018, 155:1428-35. 10.1053/j.gastro.2018.07.018

76. Kwok R, Tse YK, Wong GL, et al.: Systematic review with meta-analysis: non-invasive assessment of nonalcoholic fatty liver disease--the role of transient elastography and plasma cytokeratin-18 fragments. Aliment Pharmacol Ther. 2014, 39:254-69. 10.1111/apt.12569

77. Chalasani N, Younossi Z, Lavine JE, et al.: The diagnosis and management of nonalcoholic fatty liver disease: practice guidance from the American Association for the Study of Liver Diseases. Hepatology. 2018, 67:328-57. 10.1002/hep.29367

78. Mikolasevic I, Domislovic V, Turk Wensveen T, et al.: Screening for nonalcoholic fatty liver disease in patients with type 2 diabetes mellitus using transient elastography - a prospective, cross sectional study. Eur J Intern Med. 2020, 82:68-75. 10.1016/i.ejim.2020.08.005

79. Scorletti E, Byrne CD: Extrahepatic diseases and NAFLD: the triangular relationship between NAFLD, type 2-diabetes and dysbiosis. Dig Dis. 2016, 34:11-8. 10.1159/000447276

80. Parry SA, Hodson L: Managing NAFLD in type 2 diabetes: the effect of lifestyle interventions, a narrative review. Adv Ther. 2020, 37:1381-406. 10.1007/s12325-020-01281-6

81. Zelber-Sagi S, Godos J, Salomone F: Lifestyle changes for the treatment of nonalcoholic fatty liver disease: a review of observational studies and intervention trials. Therap Adv Gastroenterol. 2016, 9:392-407. $10.1177 / 1756283 X 16638830$

82. Browning JD, Baker JA, Rogers T, Davis J, Satapati S, Burgess SC: Short-term weight loss and hepatic 
triglyceride reduction: evidence of a metabolic advantage with dietary carbohydrate restriction. Am J Clin Nutr. 2011, 93:1048-52. 10.3945/ajcn.110.007674

83. Nseir W, Hellou E, Assy N: Role of diet and lifestyle changes in nonalcoholic fatty liver disease . World J Gastroenterol. 2014, 20:9338-44. 10.3748/wjg.v20.i28.9338

84. Zelber-Sagi S, Nitzan-Kaluski D, Goldsmith R, Webb M, Blendis L, Halpern Z, Oren R: Long term nutritional intake and the risk for non-alcoholic fatty liver disease (NAFLD): a population based study. J Hepatol. 2007, 47:711-7. 10.1016/j.jhep.2007.06.020

85. Viveiros K: The role of life style modifications in comprehensive non-alcoholic fatty liver disease treatment . Clin Liver Dis (Hoboken). 2021, 17:11-4. 10.1002/cld.1007

86. Nobili V, Carter-Kent C, Feldstein AE: The role of lifestyle changes in the management of chronic liver disease. BMC Med. 2011, 9:70. 10.1186/1741-7015-9-70

87. Tacelli M, Celsa C, Magro B, Giannetti A, Pennisi G, Spatola F, Petta S: Antidiabetic drugs in NAFLD: the accomplishment of two goals at once?. Pharmaceuticals (Basel). 2018, 11:121. 10.3390/ph11040121

88. Musso G, Cassader M, Rosina F, Gambino R: Impact of current treatments on liver disease, glucose metabolism and cardiovascular risk in non-alcoholic fatty liver disease (NAFLD): a systematic review and meta-analysis of randomised trials. Diabetologia. 2012, 55:885-904. 10.1007/s00125-011-2446-4

89. Musso G, Gambino R, Cassader M, Pagano G: A meta-analysis of randomized trials for the treatment of nonalcoholic fatty liver disease. Hepatology. 2010, 52:79-104. 10.1002/hep.23623

90. Li Y, Liu L, Wang B, Wang J, Chen D: Metformin in non-alcoholic fatty liver disease: a systematic review and meta-analysis. Biomed Rep. 2013, 1:57-64. 10.3892/br.2012.18

91. Rakoski MO, Singal AG, Rogers MA, Conjeevaram H: Meta-analysis: insulin sensitizers for the treatment of non-alcoholic steatohepatitis. Aliment Pharmacol Ther. 2010, 32:1211-21. 10.1111/j.13652036.2010.04467.x

92. Nkontchou G, Cosson E, Aout M, et al.: Impact of metformin on the prognosis of cirrhosis induced by viral hepatitis C in diabetic patients. J Clin Endocrinol Metab. 2011, 96:2601-8. 10.1210/jc.2010-2415

93. Donadon V, Balbi M, Mas MD, Casarin P, Zanette G: Metformin and reduced risk of hepatocellular carcinoma in diabetic patients with chronic liver disease. Liver Int. 2010, 30:750-8. 10.1111/j.14783231.2010.02223.x

94. Kheirandish M, Mahboobi H, Yazdanparast M, Kamal W, Kamal MA: Anti-cancer effects of metformin: recent evidences for its role in prevention and treatment of cancer. Curr Drug Metab. 2018, 19:793-7. $10.2174 / 1389200219666180416161846$

95. Sawangjit R, Chongmelaxme B, Phisalprapa P, Saokaew S, Thakkinstian A, Kowdley KV, Chaiyakunapruk N: Comparative efficacy of interventions on nonalcoholic fatty liver disease (NAFLD): a PRISMA-compliant systematic review and network meta-analysis. Medicine (Baltimore). 2016, 95:e4529. 10.1097/MD.0000000000004529

96. He L, Liu X, Wang L, Yang Z: Thiazolidinediones for nonalcoholic steatohepatitis: a meta-analysis of randomized clinical trials. Medicine (Baltimore). 2016, 95:e4947. 10.1097/MD.0000000000004947

97. Said A, Akhter A: Meta-analysis of randomized controlled trials of pharmacologic agents in non-alcoholic steatohepatitis. Ann Hepatol. 2017, 16:538-47. 10.5604/01.3001.0010.0284

98. Singh S, Khera R, Allen AM, Murad MH, Loomba R: Comparative effectiveness of pharmacological interventions for nonalcoholic steatohepatitis: a systematic review and network meta-analysis. Hepatology. 2015, 62:1417-32. 10.1002/hep.27999

99. Svegliati-Baroni G, Saccomanno S, Rychlicki C, et al.: Glucagon-like peptide-1 receptor activation stimulates hepatic lipid oxidation and restores hepatic signalling alteration induced by a high-fat diet in nonalcoholic steatohepatitis. Liver Int. 2011, 31:1285-97. 10.1111/j.1478-3231.2011.02462.x

100. Ben-Shlomo S, Zvibel I, Shnell M, et al.: Glucagon-like peptide-1 reduces hepatic lipogenesis via activation of AMP-activated protein kinase. J Hepatol. 2011, 54:1214-23. 10.1016/j.jhep.2010.09.032

101. Panjwani N, Mulvihill EE, Longuet C, et al.: GLP-1 receptor activation indirectly reduces hepatic lipid accumulation but does not attenuate development of atherosclerosis in diabetic male ApoE(-/-) mice. Endocrinology. 2013, 154:127-39. 10.1210/en.2012-1937

102. Gupta NA, Mells J, Dunham RM, Grakoui A, Handy J, Saxena NK, Anania FA: Glucagon-like peptide-1 receptor is present on human hepatocytes and has a direct role in decreasing hepatic steatosis in vitro by modulating elements of the insulin signaling pathway. Hepatology. 2010, 51:1584-92. 10.1002/hep.23569

103. Armstrong MJ, Gaunt P, Aithal GP, et al.: Liraglutide safety and efficacy in patients with non-alcoholic steatohepatitis (LEAN): a multicentre, double-blind, randomised, placebo-controlled phase 2 study. Lancet. 2016, 387:679-90. 10.1016/S0140-6736(15)00803-X

104. Honda Y, Imajo K, Kato T, et al.: The selective SGLT2 inhibitor ipragliflozin has a therapeutic effect on nonalcoholic steatohepatitis in mice. PLoS One. 2016, 11:e0146337. 10.1371/journal.pone.0146337

105. Komiya C, Tsuchiya K, Shiba K, et al.: Ipragliflozin improves hepatic steatosis in obese mice and liver dysfunction in type 2 diabetic patients irrespective of body weight reduction. PLoS One. 2016, 11:e0151511. 10.1371/journal.pone.0151511

106. Nakano S, Katsuno K, Isaji M, et al.: Remogliflozin etabonate improves fatty liver disease in diet-induced obese male mice. J Clin Exp Hepatol. 2015, 5:190-8. 10.1016/j.jceh.2015.02.005

107. Tahara A, Kurosaki E, Yokono M, et al.: Effects of SGLT2 selective inhibitor ipragliflozin on hyperglycemia, hyperlipidemia, hepatic steatosis, oxidative stress, inflammation, and obesity in type 2 diabetic mice. Eur J Pharmacol. 2013, 715:246-55. 10.1016/j.ejphar.2013.05.014

108. Hayashizaki-Someya Y, Kurosaki E, Takasu T, Mitori H, Yamazaki S, Koide K, Takakura S: Ipragliflozin, an SGLT2 inhibitor, exhibits a prophylactic effect on hepatic steatosis and fibrosis induced by choline-deficient 1-amino acid-defined diet in rats. Eur J Pharmacol. 2015, 754:19-24. 10.1016/j.ejphar.2015.02.009

109. Qiang S, Nakatsu Y, Seno Y, et al.: Treatment with the SGLT2 inhibitor luseogliflozin improves nonalcoholic steatohepatitis in a rodent model with diabetes mellitus. Diabetol Metab Syndr. 2015, 7:104. 10.1186/s13098-015-0102-8

110. Ji W, Zhao M, Wang M, et al.: Effects of canagliflozin on weight loss in high-fat diet-induced obese mice . PLoS One. 2017, 12:e0179960. 10.1371/journal.pone.0179960 
111. Jojima T, Tomotsune T, Iijima T, Akimoto K, Suzuki K, Aso Y: Empagliflozin (an SGLT2 inhibitor), alone or in combination with linagliptin (a DPP-4 inhibitor), prevents steatohepatitis in a novel mouse model of non-alcoholic steatohepatitis and diabetes. Diabetol Metab Syndr. 2016, 8:45. 10.1186/s13098-016-0169-x

112. Obara K, Shirakami Y, Maruta A, et al.: Preventive effects of the sodium glucose cotransporter 2 inhibitor tofogliflozin on diethylnitrosamine-induced liver tumorigenesis in obese and diabetic mice. Oncotarget. 2017, 8:58353-63. 10.18632/oncotarget.16874

113. Itou M, Kawaguchi T, Taniguchi E, Sata M: Dipeptidyl peptidase-4: a key player in chronic liver disease . World J Gastroenterol. 2013, 19:2298-306. 10.3748/wjg.v19.i15.2298

114. Hussain M, Majeed Babar MZ, Hussain MS, Akhtar L: Vildagliptin ameliorates biochemical, metabolic and fatty changes associated with non alcoholic fatty liver disease. Pak J Med Sci. 2016, 32:1396-401. 10.12669/pjms.326.11133

115. Balant L: Clinical pharmacokinetics of sulphonylurea hypoglycaemic drugs. Clin Pharmacokinet. 1981, 6:215-41. 10.2165/00003088-198106030-00003

116. Scott LJ: Repaglinide: a review of its use in type 2 diabetes mellitus . Drugs. 2012, 72:249-72. 10.2165/11207600-000000000-00000

117. McLeod JF: Clinical pharmacokinetics of nateglinide: a rapidly-absorbed, short-acting insulinotropic agent . Clin Pharmacokinet. 2004, 43:97-120. 10.2165/00003088-200443020-00003

118. Richardson WS, Plaisance AM, Periou L, Buquoi J, Tillery D: Long-term management of patients after weight loss surgery. Ochsner J. 2009, 9:154-9.

119. Dubasi SK, Ranjan P, Arora C, et al.: Questionnaire to assess adherence to diet and exercise advices for weight management in lifestyle-related diseases. J Family Med Prim Care. 2019, 8:689-94. 10.4103/jfmpc.jfmpc_338_18

120. Mingrone G, Panunzi S, De Gaetano A, et al.: Bariatric surgery versus conventional medical therapy for type 2 diabetes. N Engl J Med. 2012, 366:1577-85. 10.1056/NEJMoa1200111

121. Schauer PR, Kashyap SR, Wolski K, et al.: Bariatric surgery versus intensive medical therapy in obese patients with diabetes. N Engl J Med. 2012, 366:1567-76. 10.1056/NEJMoa1200225

122. Kopp HP, Kopp CW, Festa A, et al.: Impact of weight loss on inflammatory proteins and their association with the insulin resistance syndrome in morbidly obese patients. Arterioscler Thromb Vasc Biol. 2003, 23:1042-7. 10.1161/01.ATV.0000073313.16135.21

123. Miras AD, Chuah LL, Lascaratos G, et al.: Bariatric surgery does not exacerbate and may be beneficial for the microvascular complications of type 2 diabetes. Diabetes Care. 2012, 35:e81. 10.2337/dc11-2353

124. Mummadi RR, Kasturi KS, Chennareddygari S, Sood GK: Effect of bariatric surgery on nonalcoholic fatty liver disease: systematic review and meta-analysis. Clin Gastroenterol Hepatol. 2008, 6:1396-402. 10.1016/j.cgh.2008.08.012

125. Klein S, Mittendorfer B, Eagon JC, et al.: Gastric bypass surgery improves metabolic and hepatic abnormalities associated with nonalcoholic fatty liver disease. Gastroenterology. 2006, 130:1564-72. 10.1053/j.gastro.2006.01.042

126. Lee Y, Doumouras AG, Yu J, et al.: Complete resolution of nonalcoholic fatty liver disease after bariatric surgery: a systematic review and meta-analysis. Clin Gastroenterol Hepatol. 2019, 17:1040-60. 10.1016/j.cgh.2018.10.017

127. Bower G, Toma T, Harling L, et al.: Bariatric surgery and non-alcoholic fatty liver disease: a systematic review of liver biochemistry and histology. Obes Surg. 2015, 25:2280-9. 10.1007/s11695-015-1691-X

128. Padwal R, Klarenbach S, Wiebe N, et al.: Bariatric surgery: a systematic review and network meta-analysis of randomized trials. Obes Rev. 2011, 12:602-21. 10.1111/j.1467-789X.2011.00866.X

129. Lassailly G, Caiazzo R, Buob D, et al.: Bariatric surgery reduces features of nonalcoholic steatohepatitis in morbidly obese patients. Gastroenterology. 2015, 149:379-88. 10.1053/j.gastro.2015.04.014

130. Garcia-Tsao G, Bosch J, Kayali Z, et al.: Randomized placebo-controlled trial of emricasan for non-alcoholic steatohepatitis-related cirrhosis with severe portal hypertension. J Hepatol. 2020, 72:885-95. 10.1016/j.jhep.2019.12.010

131. Harrison SA, Wong VW, Okanoue T, et al.: Selonsertib for patients with bridging fibrosis or compensated cirrhosis due to NASH: Results from randomized phase III STELLAR trials. J Hepatol. 2020, 73:26-39. 10.1016/j.jhep.2020.02.027

132. Younossi ZM, Ratziu V, Loomba R, et al.: Obeticholic acid for the treatment of non-alcoholic steatohepatitis: interim analysis from a multicentre, randomised, placebo-controlled phase 3 trial. Lancet. 2019, 394:2184-96. 10.1016/S0140-6736(19)33041-7 